\title{
Follicular Conjunctivitis on Dipivefrin Therapy for Glaucoma
}

\author{
J. A. COLEIRO ${ }^{1}$ H. SIGURDSSON ${ }^{1 *}$ and J. A. LOCKYER ${ }^{2}$ \\ Dundee
}

\begin{abstract}
Summary
Dipivefrin (Propine) is an effective ocular hypotensive agent. Follicular conjunctivitis has been observed in an unexpectedly high incidence of glaucoma patients on this topical therapy. Severe discomfort was experienced by a few patients. Follicles resolved after withdrawal of this medication, but may persist or recur upon subsequent treatment with other adrenaline related compounds. Regular examination of the conjunctiva is recommended for early signs of this sideeffect, which initially may be symptom free.
\end{abstract}

The development of dipivalyl epinephrine (Dipivefrin) represents an advance in the pharmacological treatment of glaucoma. The lipophilic properties of this pro-drug enhances ocular penetration at lower concentrations than its parent compound epinephrine.$^{1}$ In concentrations of one-tenth that of epinephrine, Dipivefrin $0.1 \%$ (Propine) produces fewer side-effects, such as stinging and redness of the conjunctiva. However, an unexpectedly high number of our patients presented with conjunctival toxic effects since the product was released on the United Kingdom market in early 1984.

\section{Patients and Methods}

Thirty-two patients received twice daily topical application of Dipivefrin $0.1 \%$ (Propine) for open angle glaucoma. Thirteen were male, and nineteen were female. Their ages ranged from 37 to 86 years (mean age 68 years). Twenty-nine received medication to both eyes, while three had one eye treated.

Seven patients $(21.8 \%)$ were controlled on Dipivefrin alone; twenty-five patients (78\%) required additional Timolol $0.25 \%$ also on a twice daily regimen for the control of their glaucoma, with intraocular pressure levels below $20 \mathrm{~mm} \mathrm{Hg}$. One patient received Pilocarpine $2 \%$, and one other required systemic acetazolamide.

Three patients who had been on Dipivefrin for periods varying from 5 to 12 months presented in the summer of 1985 with severe bilateral follicular conjunctivitis with marked chemosis. Subsequently further cases came to light; Dipivefrin was suspected as being the aetiological factor. All glaucoma patients had the conjunctiva examined specifically at each clinic visit.

Thirty-two patients were identified; all had been on Dipivefrin and all showed conjunctival hyperaemia. The follicular reaction was classified as mild in $13(40.6 \%)$ moderate in 6 $(18.7 \%)$, and severe in 13 patients $(40.6 \%)$. Eyes with a severe reaction also showed subtarsal papillae. The period of time for the development of follicles on the upper and lower tarsal plate and inferior fornix varied from 5 months to 2 years from the inception

From the Departments of Ophthalmology and Pathology, Ninewells Hospital and Medical School, Dundee, Scotland.

${ }^{*}$ Currently Honorary Fellow, Professorial Unit, Moorfields Eye Hospital, London Correspondence to: J. A. Coleiro MD, FRCS, Consultant Ophthalmologist, Ninewells Hospital and Medical School, Dundee, Scotland 
of therapy (mean 12 months). Three patients receiving Dipivefrin to one eye only developed conjunctival changes in the treated eye only.

Upon withdrawal of Dipivefrin, symptoms of burning and itching improved immediately. The conjunctiva reverted to normal between 3 weeks and 14 months after cessation of treatment with Dipivefrin (mean 4.5 months). No patients were re-challenged with Dipivefrin, but two patients showed similar severe reactions upon subsequent treatment with adrenaline $1 \%$. The earliest regression was obtained in two eyes after a subconjuncival depot steroid injection was given at the conclusion of a trabeculectomy procedure; in one case this procedure was combined with cataract extraction and intraocular lens implantation.

\section{Histological features}

Biopsies of the conjunctiva were carried out which showed the following features:-

Histological examination of these conjunctival biopsies (Figs. 1, 2 and 3) revealed an intact epithelium, much of which appeared thinned, with few mucus cells; an occasional neutrophil polymorphonuclear leucocyte transgressed the epithelium.

In the subepithelial connective tissue was a marked predominantly perivascular chronic inflammatory cell infiltrate composed of lymphocytes and histiocytes with smaller number of plasma cells. No eosinophils were identified. The presence of mast cells was noted. There was variation in the density of stromal collagen with areas of fibrosis. The inflammatory infiltrate formed prominent focal aggregates but no germinal centres were identified in the many sections examined. Cell marker studies showed a predominance of T lymphocytes over B lymphocytes with only a few cells showing evidence of activation or proliferation.

Stromal vessels appeared dilated (Figs. 1 and 2). No excess of elastosis was present and no fatty deposits or granulomata were identified.

The histological features were those of chronic conjunctivitis which appeared to be predominantly $\mathrm{T}$-cell mediated response in keeping with a delayed hypersensitivity reac-

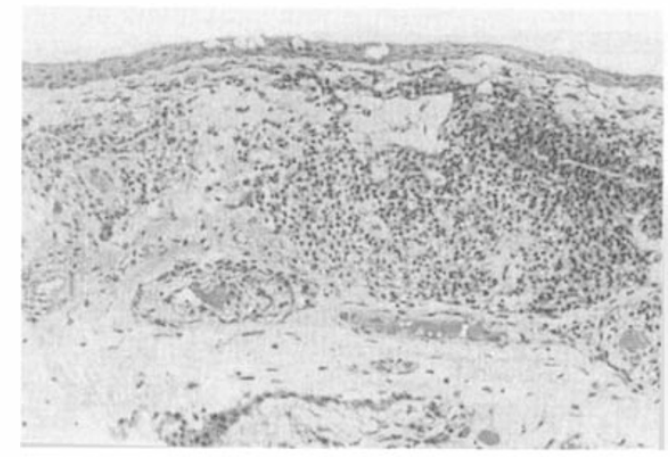

Fig. 1. Conjunctival biopsy showing focal aggregation of chronic inflammatory cell infiltate. (Haematoxylin and eosin. Plastic section). $\times 95$.

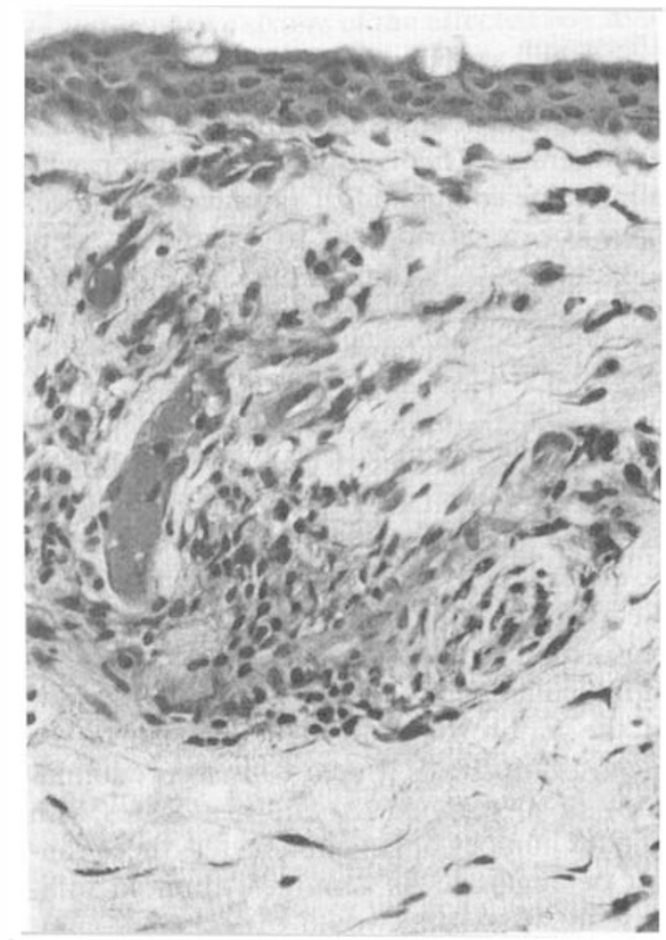

Fig. 2. Conjunctival biopsy showing a predominantly perivascular chronic inflammatory cell infiltrate composed of lymphocytes, histiocytes and smaller numbers of plasma cells. (Detail from Fig. 3) (Haematoxylin and Eosin. Plastic section $\times 250$ ).

tion. There was no evidence of true lymphoid follicle formation histologically. However, focal aggregation of the inflammatory infiltrate may account for the clinical appearance of follicular conjunctivitis. 


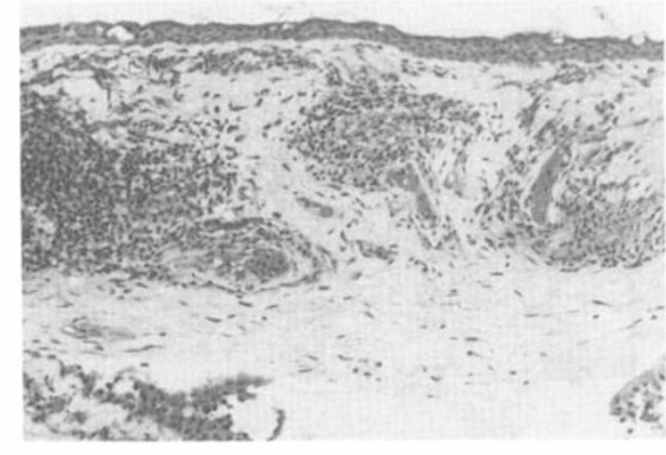

Fig. 3. Conjunctival biopsy showing focal aggregation of chronic inflammatory cell infiltrate. (Haematoxylin and Eosin. Plastic sections $\times 95$ ).

\section{Discussion}

Minor ocular discomfort such as burning, stinging and redness is frequent with topical eye therapy. This is especially common with adrenaline compounds. Conjunctival follicles have been attributed to treatment with idoxuridine, decemarium, echothiopate, physostygmine, atropine, pilocarpine, neomycin, gentamycin and chloramphenicol. ${ }^{2}$ The conjunctivitis produced by these preparations is apparently 'follicular' since proof is lacking that these drugs induce the development of true lymphoid follicles, as these lack germinal centres. Clinically, however, these reactions are indistinguishable, and Wilson ${ }^{3}$ suggests that it would be reasonable to use the traditional designation of follicular conjunctivitis. Moreover, in view of the nature of the cell population in the focal aggregates, which consist predominantly of T-lymphocytes and histiocytes with smaller number of B-lymphocytes, these cannot be regarded as genuine lymphoid follicles, in which one would expect predominantly B-lymphocytes with small numbers of macrophages and an absence of T-lymphocytes. Follicle formation may not be confirmed by immunoperoxidase staining. Bulbar follicles have been related to dipivefrin, with resolution within one month to six weeks. ${ }^{4}$ Biopsy on Liesegang's patients confirmed the presence of a lymphoid follicle. ${ }^{4}$ None of our patients had bulbar follicles; all follicles on tarsi and inferior fornix have resolved despite the continuation of other topical antiglaucoma therapy, including timolol, which also contains benzalkonium as the preservative. Our findings confirm that the duration of exposure to the drug is an important risk factor. ${ }^{5}$

Whereas large numbers of patients on dipivefrin experience only minor discomfort and conjunctival hyperaemia, severe conjunctival reactions occur more frequently than hitherto appreciated. ${ }^{6}$ Our patients were derived from an extensive geographical area, including Fife, North Angus and Perthshire, and who had obtained their supplies from various sources. Several batches returned to the manufacturer showed no abnormality in production. While dipivefrin is undoubtedly effective in lowering intraocular pressure, it is recommended that the conjunctiva is examined regularly for early changes which may progress causing severe discomfort.

We are grateful to the Medical Records Department of Ninewells Hospital, Dundee, for retrieving case notes, Mr. D. A. Orrock and Mrs. M. J. Franklin of the Dundee University Library for searches of literature, Mr. G. Coghill for Histochemistry preparations. Thanks are also due to Professor J. S. Beck and Dr. W. Guthrie for helpful advice, Mrs A. Ellingford and Mr. G. Cowper for photographic assistance, Mrs. L. Rose for secretarial help and Dr. B. Guyer, Medical Director, Allergan Laboratories.

\section{References}

${ }^{1}$ McGuinness R and Frumar KD: Timolol and dipvalyl epinephrine. Aust J Ophthalmol 1982, 10: 179-82.

2 Fraunfelder FT: Drug-induced ocular sideeffects and drug interactions. 2nd Edition. Lea and Ferbiger (Philadelphia) 1982.

3 Wilson FM: Adverse external ocular effects of topical ophthalmic medications. Surv Ophthalmol 1979, 24: 57-88.

${ }^{4}$ Liesegang TJ: Bulbar conjunctival follicles associated with Dipivefrin therapy. Ophthalmology 1985, 92: 228-33.

5 Theodore J and Leibowitz HM: External ocular toxicity of dipivalyl epinephrine. $A m \quad J$ Ophthalmol 1979, 88: 1013-6.

${ }^{6}$ Wandel T and Spinak M: Ophthalmology 1981, 88: $259-60$. 